\title{
A NUMERICAL TECHNIQUE FOR THE SOLUTION OF SINGULAR INTEGRAL EQUATIONS OF THE SECOND KIND*
}

\author{
BY \\ G. R. MILLER AND L. M. KEER \\ Northwestern University
}

\begin{abstract}
A numerical technique for the solution of singular integral equations of the second kind is presented. The technique extends the piecewise quadratic method used by Gerasoulis [6], to solve singular integral equations of the first kind. Quadrature formulae are derived in terms of infinite series and are used to reduce the integral equation to a set of linear algebraic equations. Two numerical examples are given, and the results are compared with exact solutions.
\end{abstract}

Introduction. Singular integral equations often arise in mathematical models of physical phenomena, specifically in various kinds of mixed boundary value problems. Since closed-form solutions to these integral equations are generally not available, much attention has been focused on numerical methods of solution. Generally, these methods are based on Gaussian quadrature techniques chosen such that the singular behavior of the solution is handled implicitly (see, e.g., [1]-[3]). Gerasoulis and Srivastov (see [4], [5] and [6]) present a somewhat different approach using a piecewise polynomial method. Although the singular behavior of the solution is still contained implicitly, the selection of node points can be performed arbitrarily. This has the advantages both of eliminating the need for interpolation at the endpoints and of making it possible to model with higher accuracy solutions with localized erratic behavior.

This paper extends the method used by Gerasoulis in [6] to solve singular integral equations of the first kind, to the case of singular integral equations of the second kind. This type of equation has the form

$$
R f(x)+\frac{P}{\pi} \int_{-1}^{1} \frac{f(t) d t}{t-x}+\int_{-1}^{1} K(x, t) f(t) d t=g(x), \quad-1<x<1,
$$

In Eq. (1), $R$ and $P$ are constants which may be complex in general, and the kernel $K(x, t)$ is assumed bounded for $-1 \leqslant(x, t) \leqslant 1$. The function $g(x)$ is known, and $f(x)$ is the function to be determined.

\footnotetext{
${ }^{*}$ Received August 3, 1983. The authors are grateful for support from the National Science Foundation, Grant MEA-7923261.
} 
Using results in [7], the singular behavior of the solution may be characterized by a fundamental function given by

$$
\begin{aligned}
w(t) & =(1-t)^{\alpha}(1+t)^{\beta}, \\
\alpha & =-\frac{1}{2 \pi i} \log \left(\frac{R-i P}{R+i P}\right)-N, \\
\beta & =+\frac{1}{2 \pi i} \log \left(\frac{R-i P}{R+i P}\right)-M,
\end{aligned}
$$

where $N$ and $M$ are integers determined such that the index $\kappa \equiv(\alpha+\beta)=-(N+M)$ is restricted to $-1,0,1$ to insure integrable singularities. In the case $\kappa=-1$ an additional condition is needed to render the solution unique. This condition is of the form:

$$
\int_{-1}^{1} f(x) d x=A .
$$

For $\kappa=1$ the solution must satisfy a consistency condition [7]:

$$
\int_{-1}^{1} \frac{1}{w(x)}\left[\frac{R}{P} f(x)+\frac{1}{\pi} \int_{-1}^{1} \frac{f(t) d t}{t-x}\right] d x=0 .
$$

Following $[1] f(t)$ is written as the quotient

$$
f(t)=\phi(t) / w(t) .
$$

This insures the proper behavior at the endpoints, and the unknown function is now $\phi(t)$, which is regular on $-1 \leqslant t \leqslant 1$. The function $\phi(t)$ is to be approximated by piecewise quadratic polynomials leading to a numerical collocation scheme similar to that obtained in [6].

The method is used to solve the integral equations corresponding to a rigid indenter sliding on an elastic halfplane with friction, and to the problem of an interface crack. The known exact solutions to these problems are compared with the numerical results. The method is found effective for both real and complex singularities, with an observed rate of convergence of $O\left(h^{3}\right)$. This rate of convergence is the same as in [6], and is consistent with the piecewise quadratic approximation.

Preliminary results. To develop a piecewise method it is first necessary to obtain expressions for the following indefinite integrals:

$$
\begin{aligned}
& \text { 1. } \int \frac{(t-a)^{l} d t}{(1-t)^{\alpha}(1+t)^{\beta}}, \\
& \text { 2. } \int \frac{d t}{(t-x)(1-t)^{\alpha}(1+t)^{\beta}} \text {. }
\end{aligned}
$$

This can be accomplished by expanding the weight functions according to the binomial expansion theorem:

$$
(1-t)^{-\alpha}(1+t)^{-\beta}=\sum_{n=0}^{\infty} A_{n}(a)(t-a)^{n} ; \quad|t|<1,
$$


in which

$$
A_{n}(a)=(1-a)^{-\alpha}(1+a)^{-\beta} \sum_{j=0}^{n} \frac{(\alpha)_{j}}{j !} \frac{(\beta)_{n-j}}{(n-j) !} \frac{(-1)^{n-j}}{(1-a)^{j}(1+a)^{n-j}}
$$

and following Hansen's notation [8],

$$
(\alpha)_{j} \equiv \Gamma(j+\alpha) / \Gamma(\alpha) \text {. }
$$

The constant " $a$ " will be specified later in the analysis, its choice being based on numerical considerations.

The series in (9) is absolutely convergent in the open interval $(-1,1)$. Thus, the series may be substituted into (7) and (8), and the integration performed term by term. In the case of the first integral (7), this gives

$$
\int \frac{(t-a)^{l} d t}{(1-t)^{\alpha}(1+t)^{\beta}}=(t-a)^{l+1} S_{1}(\xi)+C_{1}
$$

where

$$
S_{1}(\xi)=\sum_{n=0}^{\infty} A_{n}(a) \frac{(\xi-a)^{n}}{(n+l+1)}
$$

and $C_{1}$ is some constant. The singularities $\alpha, \beta$ are integrable, and thus the series in (11) converges over the closed interval $[-1,1]$, as can be shown by Raabe's test (see, e.g., [9]). At $|t|=1$, however, the convergence is extremely slow; too slow to be of practical use. But $S_{1}$ may be rewritten using (10) as

$$
S_{1}(1)=(1-a)^{-\alpha}(1+a)^{-\beta} \sum_{n=0}^{\infty} \frac{(-\beta)_{n}}{n !}\left(\frac{1-a}{1+a}\right)^{n} \sum_{j=0}^{\infty} \frac{(\alpha)_{j}}{j !(n+j+l+1)} .
$$

The inner sum is given by Hansen (Eq. 6.6.2) in closed form [8]:

$$
\sum_{j=0}^{\infty} \frac{(\alpha)_{j}}{j !(n+j+l+1)}=\frac{\Gamma(1-\alpha) \Gamma(n+l+1)}{\Gamma(2-\alpha-n-l)}
$$

Thus, $S_{1}(1)$ becomes

$$
S_{1}(1)=\frac{(1-a)^{-\alpha}(1+a)^{-\beta}}{(1-\alpha)} \sum_{n=0}^{\infty} \frac{(\beta)_{n}(-1)^{n}}{(2-\alpha)_{n+l}}\left(\frac{1-a}{1+a}\right)^{n} \frac{(n+l) !}{n !}
$$

which converges quite quickly for $a>0$. A similar procedure at $t=-1$ gives

$$
S_{1}(-1)=\frac{(1-a)^{-\alpha}(1+a)^{-\beta}}{(1-\beta)} \sum_{n=0}^{\infty} \frac{(\alpha)_{n}(-1)^{n}}{(2-\beta)_{n+l}}\left(\frac{1+a}{1-a}\right)^{n} \frac{(n+l) !}{n !} .
$$

Thus Eqs. (11), (14) and (15) enable the evaluation of the indefinite integral (7) on the interval $[-1,1]$.

Consider next the second integral. Proceeding in analogous fashion, the series expansion (9) is substituted into (8) and the resulting integrations performed term by term. This results in the following expression:

$$
\begin{aligned}
& \int \frac{d t}{(t-x)(1-t)^{\alpha}(1+t)^{\beta}} \\
& \quad=-(1-x)^{-\alpha}(1+x)^{-\beta} \log |t-x|+S_{2}(t, x)+C_{2} ; \quad|x|<1,
\end{aligned}
$$


where

$$
S_{2}(t, x) \equiv \sum_{n=1}^{\infty} A_{n}\left(a^{\prime}\right)\left(x-a^{\prime}\right)^{n} \sum_{j=1}^{n} \frac{1}{j} X^{j}
$$

In Eq. (16) $C_{2}$ is an arbitrary constant, $X=\left(t-a^{\prime}\right) /\left(x-a^{\prime}\right)$, and " $a^{\prime \prime}$ " is an unspecified point between -1 and 1 . As in the previous case, the infinite series converges absolutely on the closed interval $-1 \leqslant t \leqslant 1$, but very slowly at the endpoints, \pm 1 . Again, this latter difficulty can be removed by proper manipulation of the series.

To accomplish this, $S_{2}(t, x)$ is first written as

$$
S_{2}(t, x)=\sum_{j=1}^{\infty} \frac{1}{j} X^{j} \sum_{n=j}^{\infty} A_{n}\left(a^{\prime}\right)\left(x-a^{\prime}\right)^{n}
$$

which may be rewritten further as

$$
\begin{aligned}
S_{2}(t, x) & =\sum_{j=1}^{\infty} \frac{1}{j} X^{j}\left\{(1-x)^{-\alpha}(1+x)^{-\beta}-\sum_{n=0}^{j-1} A_{n}\left(a^{\prime}\right)\left(x-a^{\prime}\right)^{n}\right\} \\
& =(1-x)^{-\alpha}(1+x)^{-\beta} \log \left|\frac{x-a^{\prime}}{x-t}\right|-X \sum_{k=0}^{\infty} \frac{1}{k+1} X^{k} \sum_{n=0}^{k} A_{n}\left(a^{\prime}\right)\left(x-a^{\prime}\right)^{n} .
\end{aligned}
$$

Evaluating this expression at +1 and -1 and using (10) as before leads to

$$
\begin{aligned}
S_{2}(1, x) & =Q(1, x)-\frac{X}{1-\alpha} \sum_{k=0}^{\infty} \frac{X^{k} k !}{(2-\alpha)_{k}} \sum_{n=0}^{k}(-1)^{n} \frac{(\beta)_{n}}{n !}\left(\frac{1-a^{\prime}}{1+a^{\prime}}\right)^{n} X^{n}, \\
S_{2}(-1, x) & =Q(-1, x)-\frac{X}{1-\beta} \sum_{k=0}^{\infty} \frac{X^{k} k !}{(2-\beta)_{k}} \sum_{n=0}^{k} \frac{(\alpha)_{n}}{n !}\left(\frac{1+a^{\prime}}{1-a^{\prime}}\right)^{n} X^{n},
\end{aligned}
$$

in which

$$
Q(t, x) \equiv(1-x)^{-\alpha}(1+x)^{-\beta} \log \left[\left|x-a^{\prime}\right| /|x-t|\right] .
$$

As a final preliminary, note that in the case $a^{\prime}=x, S_{2}(t, x)$ becomes

$$
S_{2}(t, x)=\sum_{j=1}^{\infty} j^{-1} A_{j}(x)(t-x)^{j}
$$

At the endpoints the identity

$$
\sum_{j=1}^{\infty} \sum_{k=0}^{j} f(j, k)=\sum_{j=1}^{\infty} \sum_{k=0}^{\infty} f(j+k, k)+\sum_{k=0}^{\infty} f(k, k)
$$

used in conjunction with Eq. (10) makes it possible to express $S_{2}(1, x)$ in this case as

$$
S_{2}^{*}(1, x)=\sum_{n=1}^{\infty} \frac{(\beta)_{n}(-1)^{n}}{n(1-\alpha)_{n}}\left(\frac{1-x}{1+x}\right)^{n}+\sum_{j=1}^{\infty} \frac{(\alpha)_{j}}{(j) j !},
$$

and $S_{2}(-1, x)$ as

$$
S_{2}^{*}(-1, x)=\sum_{n=1}^{\infty} \frac{(\alpha)_{n}(-1)^{n}}{n(1-\beta)_{n}}\left(\frac{1+x}{1-x}\right)^{n}+\sum_{j=1}^{\infty} \frac{(\beta)_{j}}{(j) j !} .
$$


The final sum in Eqs. (23) and (24) may be effectively computed by using a truncated expansion of the digamma function (see [10]). Thus

$$
\begin{aligned}
\sum_{j=1}^{\infty} \frac{(\alpha)_{j}}{(j) j !}=\frac{1}{1-\alpha}-(1-\alpha)\{ & \sum_{k=2}^{m} Q_{k}(-1)^{k} \alpha^{k-2} \\
& \left.+\alpha^{m}(-1)^{m} \sum_{n=1}^{\infty}\left\{1 /\left[n^{m+1}(\alpha+n)\right]-1 / n^{m+2}\right\}\right\}
\end{aligned}
$$

where $Q_{k} \equiv \sum_{j=1}^{\infty} j^{-k}$. The values of the sums of reciprocal powers $Q_{k}$ may be found tabulated in [11]. The calculations presented later use $m=6$.

The preceding preliminary results provide the necessary expressions to evaluate the indefinite integral (8) over the closed interval $-1 \leqslant t \leqslant 1$.

General quadrature formula. Following [6], the interval $[-1,1]$ is partitioned into a series of subintervals over which the unknown function, $\phi$, and the kernel function are approximated by quadratic Lagrange interpolation polynomials. Thus, divide the interval $[-1,1]$ into $N$ subintervals, and define integration points, $t_{i}$, at the ends and midpoint of each subinterval. Further define collocation points $x_{j}=\left(t_{j}+t_{j+1}\right) / 2, j=1,2 N$. Over each subinterval $k\left(t_{2 k-1} \leqslant t \leqslant t_{2 k+1}\right)$ approximate $\phi(t)$ by

$$
\begin{aligned}
\phi(t) \approx & \phi_{2 k-1}\left[\left(t-t_{2 k}\right)^{2} / 2 h_{k}^{2}-\left(t-t_{2 k}\right) / 2 h_{k}\right]+\phi_{2 k}\left[1-\left(t-t_{2 k}\right)^{2} / h_{k}^{2}\right] \\
& +\phi_{2 k+1}\left[\left(t-t_{2 k}\right)^{2} / 2 h_{k}^{2}+\left(t-t_{2 k}\right) / 2 h_{k}\right]
\end{aligned}
$$

where $\phi_{j} \equiv \phi\left(t_{j}\right)$ and $h_{k} \equiv\left(t_{2 k+1}-t_{2 k-1}\right) / 2$. The product $\phi(t) K(x, t)$ may be approximated in analogous fashion.

The integrals over the interval $[-1,1]$ in (1) can now be approximated by the sums of integrals over the sub-intervals. Define

$$
\begin{aligned}
I_{1}(l, k) & \equiv \int_{t_{2 k-1}}^{t_{2 k+1}} \frac{\left(t-t_{2 k}\right)^{l} d t}{(1-t)^{\alpha}(1+t)^{\beta}}, \\
I_{2}(x, k) & \equiv \int_{t_{2 k-1}}^{t_{2 k+1}} \frac{d t}{(t-x)(1-t)^{\alpha}(1+t)^{\beta}}
\end{aligned}
$$

and note that these integrals may be evaluated from the expressions obtained earlier. The integals in Eq. (1) are then approximated by

$$
\int_{-1}^{1} \frac{f(t) d t}{t-x} \approx \sum_{i f=1}^{2 N+1} \phi_{i} w_{i}(x)
$$

and

$$
\int_{-1}^{1} K(x, \xi) f(\xi) d \xi \approx \sum_{i=1}^{2 N+1} K\left(x, t_{i}\right) \phi_{i} v_{i}
$$


in which the variable weights are given by

$$
w_{i}(x)= \begin{cases}W L\left(\frac{i+1}{2}, x\right) & i=1, \\ W L\left(\frac{i+1}{2}, x\right)+W R\left(\frac{i-1}{2}, x\right) & i \text { odd } \\ W M\left(\frac{i}{2}, x\right) & i \text { even, } \\ W R\left(\frac{i-1}{2}, x\right) & i=2 N+1\end{cases}
$$

and the constant weights by

$$
v_{i}= \begin{cases}V L\left(\frac{i+1}{2}\right) & i=1, \\ V L\left(\frac{i+1}{2}\right)+V R\left(\frac{i-1}{2}\right) & i \text { odd, } \\ V M\left(\frac{i}{2}\right) & i \text { even, } \\ V R\left(\frac{i-1}{2}\right) & i=2 N+1,\end{cases}
$$

The functions used above are as follows:

$$
\begin{aligned}
W L(r, x)= & \frac{1}{2}\left[q^{2}(r, x)-q(r, x)\right] I_{2}(x, r)-[1-q(r, x)] I_{1}(0, r) / 2 h_{r} \\
& +I_{1}(1, r) / 2 h_{r}^{2}, \\
W M(r, x)= & {\left[1-q^{2}(r, x)\right] I_{2}(x, r)-\left[q(r, x) I_{1}(0, r)+I_{1}(1, r) / h_{r}\right] / h_{r}, } \\
W R(r, x)= & {\left[q^{2}(r, x)+q(r, x)\right] I_{2}(x, r) / 2+[1+q(r, x)] I_{1}(0, r) / 2 h_{r} } \\
& +I_{1}(1, r) / 2 h_{r}^{2},
\end{aligned}
$$

with $q(r, x) \equiv\left(x-t_{2 r}\right) / h_{r}$, and

$$
\begin{aligned}
& V L(r)=\left[I_{1}(2, r)-I_{1}(1, r)\right] / 2 h_{r}, \\
& V M(r)=I_{1}(0, r)-I_{1}(2, r) / h_{r}^{2}, \\
& V R(r)=\left[I_{1}(2, r)+I_{1}(1, r)\right] / 2 h_{r} .
\end{aligned}
$$

Equations (29) and (30) thus make it possible to reduce Eqs. (1) and (4) to the following system of linear algebraic equations (assuming here $\kappa=-1$; the other cases can be handled in analogous fashion):

$$
\begin{gathered}
\frac{R}{\left(1-x_{j}\right)^{\alpha}\left(1+x_{j}\right)^{\beta}} \sum_{m=1}^{3} B_{m} \phi_{I+m}+\sum_{i=1}^{2 N+1}\left[\frac{p}{\pi} w_{i}\left(x_{j}\right)+K\left(x_{j}, t_{i}\right) v_{i}\right] \phi_{i}=g\left(x_{j}\right) \\
j=1,2 N \\
\sum_{i=1}^{2 N+1} v_{i} \phi_{i}=A
\end{gathered}
$$


The first sum on the left-hand side of Eq. (35) is needed to transform the values of $\phi$ evaluated at the collocation points, $x_{j}$, to the values of $\phi$ at the integration points, $t_{i}$. The terms in this sum may be written

$$
\begin{aligned}
& B_{1}=\left\{\begin{array}{rr}
3 / 8, & j \text { odd }, \\
-1 / 8, & j \text { even, }
\end{array}\right. \\
& B_{2}=3 / 4 \quad \text { all } j, \\
& B_{3}=\left\{\begin{array}{rr}
-1 / 8, & j \text { odd }, \\
3 / 8, & j \text { even, }
\end{array}\right.
\end{aligned}
$$

and

$$
I= \begin{cases}j-1, & j \text { odd } \\ j-2, & j \text { even }\end{cases}
$$

This gives $2 N+1$ equations with which to solve for the $2 N+1$ values of the unknown function, $\phi_{j}$.

The convergence of the method can be proved, but as the proof is entirely analogous to that in [6], it is omitted here.

Numerical examples and conclusions. Consider the problem of a rigid punch sliding with friction on a half-plane. The integral equation corresponding to this is

$$
-\eta\left(\frac{\kappa-1}{\kappa+1}\right) f(x)+\frac{1}{\pi} \int_{-1}^{1} \frac{f(t)}{t-x}=K_{1} g^{\prime}(x)
$$

with the condition (assuming sharp corners)

$$
\int_{-1}^{1} f(t) d t=L
$$

In (38), $\kappa$ and $\mu$ are elastic constants, $\eta$ is the coefficient of friction, $L$ is the external load, $g(x)$ represents the shape of the indenter, and $K_{1}=4 \mu(1-i \eta) /(\kappa+1)$. For $K_{1} g^{\prime}(x)=$ $-x$, the solution to (39) is

$$
f(x)=\left\{\frac{L}{\pi}+\left[x^{2}+(1-2 \alpha) x+2 \alpha(\alpha-1)\right]\right\} \frac{\sin \pi \alpha}{(1-x)^{\alpha}(1+x)^{\beta}}
$$

with

$$
\alpha=\frac{1}{\pi} \tan ^{-1}\left[\frac{(\kappa+1)}{\eta(\kappa-1)}\right], \quad \beta=1-\alpha .
$$

The solution to (38) was computed numerically with $\alpha=0.34, \beta=0.66$, and $L=$ $\pi / \sin \alpha \pi$, using the piecewise quadratic method. The results of this computation are compared with the exact solution in Table 1. (Note that the singular part of the solution has been factored out.) The results show that the method is exact in this case as it should be, since the analytic solution is quadratic. 


\begin{tabular}{|l|l|l|}
\hline$t$ & \multicolumn{1}{|c|}{$n=9$} & Exact \\
\hline-1.0 & 1.202602104 & 1.202602104 \\
-0.75 & 0.889322466 & 0.889322466 \\
-0.50 & 0.685581163 & 0.685581163 \\
-0.25 & 0.591378195 & 0.591378195 \\
0.0 & 0.606713562 & 0.606713562 \\
0.25 & 0.731587264 & 0.731587264 \\
0.50 & 0.96599301 & 0.965999301 \\
0.75 & 1.309949673 & 1.309949673 \\
1.0 & 1.76343838 & 1.76343838 \\
\hline
\end{tabular}

TABLE 1

\begin{tabular}{|l|l|l|l|}
\hline \multicolumn{1}{|c|}{$t$} & \multicolumn{1}{|c|}{$n=17$} & \multicolumn{1}{c|}{$n=33$} & \multicolumn{1}{c|}{ Exact } \\
\hline 0.0 & $\left(0.02653,0\left(10^{-14}\right)\right)$ & $\left(0.02623,0\left(10^{-14}\right)\right)$ & $(0.02619,0.0)$ \\
0.25 & $(0.01682,-.15533)$ & $(.01653,-0.15531)$ & $(0.01649,-0.15531)$ \\
0.5 & $(-0.01229,-0.17939)$ & $(-0.01258,-0.17934)$ & $(-0.01262,-0.17934)$ \\
0.75 & $(-0.06082,0.05907)$ & $(-0.06109,0.05917)$ & $(-0.06113,0.05918)$ \\
1.0 & $(-0.12886,0.69074)$ & $(-0.12903,0.69143)$ & $(-0.12906,0.69153)$ \\
\hline
\end{tabular}

TABLE 2

Consider next the mathematical representation of an interface crack. Here the integral equation is given by

$$
\begin{aligned}
& {\left[\left(\kappa_{1}-1\right) / 4 \mu_{1}-\left(\kappa_{2}-1\right) / 4 \mu_{2}\right] f(x)} \\
& \quad+\frac{1}{\pi i}\left[\left(\kappa_{1}+1\right) / 4 \mu_{1}+\left(\kappa_{2}+1\right) / 4 \mu_{2}\right] \int_{-1}^{1} \frac{f(t) d t}{t-x}=K_{2} p(x)
\end{aligned}
$$

subject to

$$
\int_{-1}^{1} f(t) d t=0
$$

Again, $\kappa$ and $\mu$ are elastic constants with subscripts referring to material 1 and $2, p(x)$ corresponds to the applied stress distribution in the crack, and

$$
K_{2}=\left(2 \mu_{1} \mu_{2}\right)^{-2}\left(\kappa_{1} \mu_{2}+\mu_{1}\right)\left(\kappa_{2} \mu_{1}+\mu_{2}\right) .
$$

For $K_{2} p(x)=x^{2}$, the solution to (41) is

$$
f(x)=\frac{1}{Q}\left[x^{3}+(1-2 \alpha) x^{2}+2 \alpha(\alpha-1) x-\frac{\alpha}{3}\left(4 \alpha^{2}-6 \alpha+2\right)\right] \frac{\sin \pi \alpha}{(1-x)^{\alpha}(1+x)^{\beta}}
$$

in which

$$
\begin{aligned}
& \alpha=\frac{1}{2}-\frac{i}{2 \pi} \log \left|\frac{\kappa_{1} \mu_{2}+\mu_{1}}{\kappa_{2} \mu_{1}+\mu_{2}}\right| ; \quad \beta=1-\alpha, \\
& Q=-i\left[\left(\kappa_{1}+1\right) / 4 \mu_{1}+\left(\kappa_{2}+1\right) / 4 \mu_{2}\right] .
\end{aligned}
$$


The piecewise quadratic method was used to compute numerical solutions to Eqs. (41) and (42) with $\kappa_{2} p(x)=x^{2}, \mu_{1} / \mu_{2}=0.10, \kappa_{1}=1.6$ and $\kappa_{2}=2.0$. The results of these calculations are presented in Table 2, where again the singular part of the solution has been factored out. It can be seen that the method is applicable in the case of complex singularities. The rate of convergence can be calculated from Table 2 using the formula

$$
-p \log 2=\log \left|\frac{e_{2 n}}{e_{n}}\right| ; \quad e_{n}=f(x)-f_{n}(x)
$$

where the rate is defined by $O\left(h^{p}\right)$. Since the approximation is quadratic, the rate should be $O\left(h^{3}\right)$, which indeed is the value calculated using Eq. (45) and Table 2.

In the event that one value of $\phi$ is of particular importance, unequal spacing of the integration intervals can be used to obtain higher local accuracy without introducing additional integration points. Thus, in computing stress intensity factors, it is possible to obtain somewhat better accuracy than is immediately apparent from Table 2 . In practice one to two additional significant figures can be gained, depending on the behavior of the kernel and the right-hand side of the equation, and on the number of points being used.

It is evident, then, that the piecewise method can be used to solve equations of the type (1), including the case where complex singularities arise. Since the quadrature formulae were derived independently of the exact form of the integral equation, it would appear in addition that the expressions presented here could be useful in solving other classes of singular integral equations in which general singularities appear in the solution. Finally, extending the quadrature formulae to higher order piecewise approximations (i.e., cubic, quartic, etc.) could be performed fairly easily, were it so desired.

Appendix. The largest proportion of computer time is spent computing the values $I_{1}(l, k)$ and $I_{2}\left(x_{j}, k\right)$, and so it is most efficient to compute these values once and store them in arrays. Through proper choice of the expansion points $a$ and $a^{\prime}$, the various sums derived earlier can be made to converge quite rapidly. For $I_{1}(l, k)$ it has been assumed implicitly that $a=t_{2 k}$. This gives good convergence, and leads to the following expressions for $I_{1}$ :

$$
I_{1}(l, k)= \begin{cases}2 \sum_{n=n_{b}, n_{b+2}, n_{b+4}, \ldots}^{\infty} \frac{A_{n}\left(t_{2 k}\right) h_{k}^{n+l+1}}{(n+l+1)} ; & k \neq 1, N \\ \sum_{n=0}^{\infty} \frac{A_{n}\left(t_{2}\right) h_{1}^{n+l+1}}{n+l+1}-S_{1}(-1) ; & k=1, \\ S_{1}(1)-\sum_{n=0}^{\infty} \frac{A_{n}\left(t_{2 k}\right) h_{N}^{n+l+1}}{n+l+1} ; & k=N ;\end{cases}
$$

where

$$
n_{b}= \begin{cases}0, & l=0,2 \\ 1, & l=1\end{cases}
$$


Computing $I_{2}\left(x_{j}, k\right)$ is more complicated as the best choice of $a^{\prime}$ depends on $x_{j}$. Introducing the notation

$$
W F\left(x, p_{1}, p_{2}\right)=\int_{p_{1}}^{p_{2}} \frac{d t}{(t-x)(1-t)^{\alpha}(1+t)^{\beta}} .
$$

$I_{2}$ may be expressed as follows:

$$
I_{2}\left(x_{j}, k\right)= \begin{cases}W F\left(x_{1},-1, t_{m}\right)+W F\left(x_{1}, t_{m}, t_{3}\right), & j, k=1, \\ W F\left(x_{N}, t_{2 N-1}, t_{m}\right)+W F\left(x_{N}, t_{m}, 1\right), & j, k=N, \\ W F\left(x_{N}, t_{2 k-1}, t_{2 k+1}\right), & \text { otherwise }\end{cases}
$$

The point $t_{m}$ is an intermediate point chosen such that convergent forms of the sums are obtained. Thus,

$$
t_{m}= \begin{cases}\frac{1}{2}\left(x_{1}+t_{2}\right), & j, k=1, \\ \frac{1}{2}\left(t_{2 N}+x_{N}\right), & j, k=N .\end{cases}
$$

Explicit expressions for $W F$ are summarized below:

Case $1 .\left|p_{1}\right| \neq 1,\left|p_{2}\right| \neq 1 ; x<p_{1}$ or $x>p_{2}$.

$$
W F\left(x, p_{1}, p_{2}\right)=2 \sum_{n=0,2,4 \ldots}^{\infty} \frac{1}{(n+1)}\left(\frac{p_{2}-a^{\prime}}{x-a^{\prime}}\right)^{n+1} \sum_{s=0}^{n} A_{s}\left(a^{\prime}\right)\left(x-a^{\prime}\right)^{s}
$$

with $a^{\prime}=\left(p_{1}+p_{2}\right) / 2$

Case 2. $\left|p_{1}\right| \neq 1,\left|p_{2}\right| \neq 1 ; p_{1}<x<p_{2}$.

$$
\begin{aligned}
W F\left(x, p_{1}, p_{2}\right)= & \frac{1}{(1-x)^{\alpha}(1+x)^{\beta}} \log \left|\frac{p_{1}-x}{p_{2}-x}\right| \\
& -\sum_{n=1}^{\infty} \frac{A_{n}(x)}{n}\left[\left(p_{2}-x\right)^{n}-\left(p_{1}-x\right)^{n}\right] .
\end{aligned}
$$

Case 3. $p_{1}=-1 ; \frac{1}{2}\left(p_{1}+p_{2}\right)<x<-\frac{1}{4}$.

$$
\begin{aligned}
W F\left(x, p_{1}, p_{2}\right)= & \frac{1}{(1-x)^{\alpha}(1+x)^{\beta}} \log \left|\frac{-1-x}{p_{2}-x}\right|+S_{2}^{*}(-1) \\
& -\sum_{n=1}^{\infty} \frac{A_{n}(x)}{n}\left(p_{2}-x\right)^{n} .
\end{aligned}
$$

Case 4. $p_{2}=1 ; \frac{1}{4}<x<\frac{1}{2}\left(p_{1}+p_{2}\right)$.

$$
\begin{aligned}
W F\left(x, p_{1}, p_{2}\right)= & \frac{1}{(1-x)^{\alpha}(1+x)^{\beta}} \log \left|\frac{p_{1}-x}{1-x}\right|-S_{2}^{*}(1) \\
& +\sum_{n=1}^{\infty} \frac{A_{n}(x)}{n}\left(p_{1}-x\right)^{n} .
\end{aligned}
$$


Case 5. $p_{1}=-1 ;-\frac{1}{4} \leqslant x<1$.

$$
\begin{aligned}
W F\left(x, p_{1}, p_{2}\right)= & \sum_{m=0}^{\infty} \frac{1}{2^{m+1}} \sum_{n=0}^{m} \frac{(-m)_{n}(-1)^{n}}{n !(n+1)}\left(\frac{p_{2}-a^{\prime}}{x-a^{\prime}}\right) \sum_{p=0}^{m} A_{p}\left(a^{\prime}\right)\left(x-a^{\prime}\right)^{p} \\
& -S_{2}(-1)
\end{aligned}
$$

with $a^{\prime}=\left(p_{1}+p_{2}\right) / 2$.

Case 6. $p_{2}=1 ;-1<x \leqslant \frac{1}{4}$.

$$
\begin{aligned}
W F\left(x, p_{1}, p_{2}\right)= & S_{2}(1)-\sum_{m=0}^{\infty} \frac{1}{2^{m+1}} \sum_{n=0}^{m} \frac{(-m)_{n}(-1)^{n}}{n !(n+1)}\left(\frac{p_{1}-a^{\prime}}{x-a^{\prime}}\right)^{n} \\
& \cdot \sum_{p=0}^{n} A_{p}\left(a^{\prime}\right)\left(x-a^{\prime}\right)^{p}
\end{aligned}
$$

with $a^{\prime}=\left(p_{1}+p_{2}\right) / 2$.

Note. The sums in cases 5 and 6 correspond to Euler transformations [9] of the sum in Eq. (19).

\section{REFERENCES}

[1] F. Erdogan and G. Gupta, On the numerical solution of singular integral equations, Quart. Appl. Math. 30, 525-534 (1972)

[2] S. Krenk, On quadrature formulas for singular integral equations of the first and second kind, Quart. Appl. Math. 33, 225-232 (1975)

[3] P. S. Theocaris, and N. I. Iokimidis, Numerical integration methods for the solution of singular integral equations, Quart. Appl. Math. 35, 173-182 (1977)

[4] A. Gerasoulis, and R. P. Srivastav, $A$ method for the numerical solution of singular integral equations with a principal value integral, Int. J. of Engn. Sci. 19, 1293-1293 (1981)

[5] A. Gerasoulis, Piecewise polynomial approximations in the solution of singular integral equations, in Advances in Computer Methods for Partial Differential Equations, R. Vichnevetsky and R. S. Stepleman, eds., MACS 1981, 386-390

[6] A. Gerasoulis, The use of piecewise quadratic polynomials for the solution of singular integral equations of Cauchy type, Comp. and Math. with Appl. 8, 15-22 (1982)

[7] N. I. Muskhelishvili, Singular integral equations, 2nd ed., P. Noordhoff, Groningen, 1953

[8] E. R. Hansen, $A$ table of series and products, Prentice-Hall, Ind., Englewood Cliffs, New Jersey, 1975

[9] K. Knopp, Theory and application of infinite series, Blackie and Son Ltd, London, 1928

[10] C. Jordan, Calculus of finite differences, 3rd ed., Chelsea Publishing Co. New York, 1965, 327

[11] W. H. Beyer, ed., CRC standard mathematical tables, 25th ed., CRC Press, Inc., Boca Raton, Florida, 1973 